\title{
Measuring dietary fatty acid intake: validation of a food-frequency questionnaire against $7 \mathrm{~d}$ weighed records
}

\author{
Emma Broadfield*, Tricia McKeever, Andrew Fogarty and John Britton \\ Division of Respiratory Medicine, University of Nottingham, UK
}

(Received 2 September 2002 - Revised 17 February 2003 - Accepted 3 March 2003)

\begin{abstract}
There are few validated methods of measuring dietary fatty acid intake that are suitable for epidemiological research. The purpose of the present study was to develop and validate a food-frequency questionnaire (FFQ) developed to measure individual dietary fatty acid intakes against $7 \mathrm{~d}$ weighed dietary records, in a sample of thirty-one healthy adult volunteers. The FFQ was based on a previously validated questionnaire (DIETQ; Tinuviel Software, Warrington, Ches., UK), adapted to include greater detail on those foods from which the majority of dietary fatty acids are obtained. The FFQ and weighed records were analysed using food nutrient data from McCance and Widdowson's Food Composition Tables, supplemented with a food fatty acid content database (Foodbase, London, UK). Results from the two dietary assessment methods were compared by correlation coefficients and limits of agreement. The mean intake of individual fatty acids tended to be lower when assessed by FFQ. Correlation coefficients comparing unadjusted individual fatty acid intakes assessed by FFQ and weighed records ranged from 0.29 for $18: 1 n-9$ to 0.71 for $20: 4 n-6$. Adjusting for energy intake tended to increase the correlation coefficients between saturated fatty acids and decrease those between unsaturated fatty acids. In conclusion, this food-frequency method provides reliable estimates of dietary intake of many individual fatty acids for use in epidemiological studies.
\end{abstract}

Nutritional assessment: Fatty acids: Epidemiology: Diet

Fatty acids (FA) have a central role in the structure and function of cell membranes, and as a result there has been interest in the possible role of FA in the aetiology of several diseases (Horrobin, 1987; Simopoulos, 1996; Belluzzi et al. 2000) including asthma (Fogarty \& Britton, 2000; Schwartz, 2000), allergy (Black, 1999) and cardiovascular disease (Leaf \& Weber, 1988). Moreover, changes in dietary intake of FA have been shown to alter the composition of those in cell membranes, and consequently the production of their metabolites and related cell mediators (Lee \& Austen, 1986; Endres et al. 1989; Berlin et al. 1998).

For some of these diseases, such as allergy and asthma, prevalences have been increasing over the last few decades (Vollmer et al. 1998; Broadfield et al. 2002). Furthermore, over a similar period of time there have been large changes in dietary intake of many food groups and their composite nutrients in the developed world. Although there has been little change in the proportion of total energy intake obtained from fat in the UK population, there has been a marked decrease in the intake of saturated fat and an increase in intake of polyunsaturated fat (Ministry of Agriculture, Fisheries and Food, 2001a). At the present time, little data is available for the use of individual types of cooking oils in the UK, but data from the National Food
Survey shows that the average person consumes $39 \mathrm{~g}$ butter, $21 \mathrm{~g}$ margarine, $6 \mathrm{~g}$ lard, $47 \mathrm{~g}$ vegetable oil and $20 \mathrm{~g}$ low-fat spreading fat per week (Ministry of Agriculture, Fisheries and Food, 2001b). Nevertheless, investigation of the relationship between dietary FA and disease in the general population has been limited by lack of methods suitable for population studies of measuring dietary FA intakes.

Food-frequency questionnaires (FFQ) have become an increasingly popular method of assessing diet in epidemiological studies since their introduction in the 1950s, as they are a relatively inexpensive tool of assessment and tend to be more accessible than other dietary assessment methods such as weighed records. However, the methods available to epidemiological studies evaluating the role of dietary fats on disease have to date tended to estimate intakes of the major classes of fats, such as saturated $v$. polyunsaturated fats, but not individual FA, due to the limited availability of FA food composition data. In the present study we have adapted an established semi-quantitative FFQ used in epidemiological studies to assess dietary FA consumption in addition to other more commonly measured nutrients, by linking the FFQ to a separate FA database and then validating the questionnaire data in relation to intakes of FA estimated from $7 \mathrm{~d}$ weighed records.

\footnotetext{
Abbreviations: FA, fatty acid; FFQ, food-frequency questionnaire.

* Corresponding author: Dr Emma Broadfield, fax +44 115840 4771, email emma@ejcbroad.fsnet.co.uk
} 


\section{Methods}

\section{Study population}

Volunteers recruited from members of staff and research students from the Division of Respiratory Medicine (Nottingham, UK) completed a semi-quantitative FFQ and $7 \mathrm{~d}$ weighed record between January 2000 and July 2001. The participants kept the $7 \mathrm{~d}$ weighed record within 2 weeks of completing the questionnaire; the sequence in which the two dietary assessments were completed was not stipulated by the study design but by convenience to the participant.

\section{Semi-quantitative food-frequency questionnaire}

We adapted the DietQ semi-quantitative FFQ (Tinuviel Software, Warrington, Ches., UK) (Burr et al. 1989) by expanding the questions to include greater detail of the food items (Table 1) from which the majority of dietary FA are obtained. Specifically, we included questions pertaining to whether dairy products consumed were normal- or low-fat products, the types and quantities of spreading and cooking fats or oils used and whether fat was trimmed from the meat routinely. Participants were asked to record how often on average they had eaten the individual food items each week over the previous month, and were given nine possible answers from once to seven times per week, every 2 to 3 weeks, or rarelynever. Each food item was assigned a portion size using commonly used units where possible (for example, a slice of bread or teaspoon of sugar) or by using average portion sizes from a previous study of weighed records (Crawley, 1994).

The additional food items were chosen by initially identifying important dietary sources of FA, and then by excluding those foods that were infrequently consumed in

Table 1. Food items assessed in the food-frequency questionnaire

\begin{tabular}{|c|c|c|}
\hline Bread and rolls & Carrots & Dressing fat \\
\hline White bread & Parsnips & Nuts and pulses \\
\hline Brown bread & Baked beans & Peanut butter \\
\hline Wholemeal bread & Onions & Brazil nuts \\
\hline Chapatis and Paratha & Spaghetti and other pasta & Peanuts \\
\hline Naan & Rice & Almonds \\
\hline Crispbread & Spring greens & Hazelnuts \\
\hline Breakfast cereals & Green beans & Walnuts \\
\hline Cornflakes & Brussel sprouts & Coconut (fresh) \\
\hline Frosties & Cauliflower & Coconut (desiccated) \\
\hline Weetabix & Broccoli & Lentils \\
\hline Allbran & Spinach & Chick peas \\
\hline Muesli & Tomatoes & Hummus \\
\hline Porridge & Coleslaw & Puddings and sweet snacks \\
\hline Meats & Canned sweetcorn & Plain biscuits \\
\hline Beef & Canned mixed vegetables & Chocolate biscuits \\
\hline Minced beef & Other green vegetables and salads & Sandwich cream biscuits \\
\hline Lamb & Avocado pears & Other sweet biscuits \\
\hline Pork & Olives in oil & Chocolate bars \\
\hline Bacon & Olives in brine & Sweets \\
\hline Ham & Pre-packed salads & Crisps \\
\hline Chicken & Eggs, milk products, sauces, soups & Cereal bars \\
\hline Offal & Eggs & Fruit cake \\
\hline Canned meat & Milk & Sponge cake \\
\hline Meat pies & Cream & Fruit or jam tart \\
\hline Vegetarian pies and pasties & Yoghurt & Fruit pies \\
\hline Sausages & Milk pudding & Pastries \\
\hline Pre-packed prepared foods & Custard & Sponge puddings \\
\hline Fish & Ice cream & Drinks \\
\hline Fish fingers & Full-fat cheese & Tea \\
\hline Battered fish & Medium-fat cheese & Coffee \\
\hline White fish & Reduced-fat cheese & Decaffinated coffee \\
\hline Kippers & Cottage cheese & Cocoa \\
\hline Pilchards & Vegetarian cheese & Drinking chocolate \\
\hline Tuna & Quiche & Other bedtime drinks \\
\hline Shellfish & Salad cream & Fruit juice \\
\hline Fruit & Cooking sauce & Squash \\
\hline Canned & Pesto sauce & Carbonated drinks \\
\hline Apples & Packet soups & Beer \\
\hline Pears & Meat soups & Wine \\
\hline Oranges and grapefruit & Vegetable soups & Sherry \\
\hline Bananas & Fats & Spirits \\
\hline Vegetables & Spreading fat & Additional foods and vitamins \\
\hline Potatoes (boiled) & Shallow-fry fat & Eat out \\
\hline Potatoes (roasted) & Deep-fry fat & British takeaway \\
\hline Chips (oven or microwave) & Roasting fat & Chinese takeaway \\
\hline Chips (takeaway or deep fried) & Baking fat & Indian takeaway \\
\hline Peas & Sauces fat & Vitamins and minerals \\
\hline
\end{tabular}


the UK population. The final questionnaire included 129 food items, and two further questions relating to food eaten outside the home and vitamin supplementation. Questionnaire responses were then analysed using the customised DietQ software (Tinuviel Software), to which data on FA had been added from the Foodbase databank (The Institute of Brain Chemistry and Human Nutrition, University of North London, London, UK). The Foodbase FA databank was generated by repeated analysis by GC of a wide range of meals and food items. The Foodbase FA databank and DietQ were interfaced using the food item codes in McCance and Widdowson's The Composition of Foods (Holland et al. 1991).

\section{$7 d$ Weighed record}

After brief instructions, each participant was supplied with cooking scales accurate to $1 \mathrm{~g}$ (Soehnle vita 2; CMS Weighing Equipment Ltd, London, UK) and a blank diary, and asked to enter the names and weights of all food items and drinks consumed over a $7 \mathrm{~d}$ consecutive period. This included types and brands of spreading fats and cooking oils. Participants were requested not to make any alterations to their normal diets during this period. For food and drink consumed outside the home the participants were asked to enter as much detail as possible about the items and estimates of the portion sizes. Any questions or incomplete information were verified with the study subjects by the investigating researcher. The data were entered from the paper diaries on to a customised computer programme (WISP; Tinuviel Software) also adapted to incorporate the Foodbase data. A wide range of composite meals was included in the analysis program, but where an exact match was not found a similar meal was substituted.

\section{Statistical methods}

Bias and limits of agreement for the two methods were estimated as described by Bland \& Altman (1986). For those FA with a normal distribution of the difference in measured intake, and with no relationship between the difference in FA intake and mean FA intake as measured by the two methods, the bias is summarised by the mean difference and the limits of agreement by the mean value $\pm 1.96 \mathrm{sD}$ of the difference. We were unable to normalise the distribution of difference in intakes of dietary assessment method by transformation for some FA, and have therefore given the limits of agreement produced by the method described above as an approximation. For those FA that demonstrated a relationship between the difference and the mean of the two dietary assessment methods we log-transformed the data, and from this calculated a dimensionless ratio of the limits of agreement (Bland \& Altman, 1986). The two dietary assessment methods were compared using the paired $t$ test if the distribution of difference in intake between the methods was normal, and by the Wilcoxon signed rank test if not. The FA levels were transformed as necessary to normalise their distributions, and adjusted for total energy intake using energy-adjusted intake computed as the residuals from regression models, with energy intake as the independent variable and the FA as the dependent variable (Willett \& Stampfer, 1986; Willett, 1990a). Where the FA intakes could be transformed to a normal distribution we used the Pearson correlation coefficient to assess the correlation between the two dietary assessment methods; otherwise the Spearman correlation coefficient was used. Agreement on relative nutrient intake was assessed by ranking and aggregation into quartiles of intake. All analyses were carried out using the STATA 7.0 statistical package (Stata Corporation, College Station, TX, USA). Statistical significance was defined as a $P$ value $<0 \cdot 05$.

\section{Results}

The mean age of the thirty-one participants was 42.2 (SD 10.8) years and fifteen (48\%) were male. The level of education attained by those participating in the study was high with eighteen $(58 \%)$ completing further education, three $(10 \%)$ remaining at school until the age of 18 years, four $(13 \%)$ remaining at school until the age of 16 years and six (19\%) leaving school before the age of 16 years. The mean energy intake of the participants from the FFQ and weighed records were 8088 (range 4033-13259) and 9180 (range 4853-14104) kJ/d respectively, and the mean intake of FA 62.7 (range 17.8-144.7) and $64.2(27 \cdot 0-110 \cdot 8) \mathrm{g} / \mathrm{d}$ respectively.

The mean nutrient intakes obtained from both dietary methods were similar (Table 2), although apart from oleic $(18: 1 n-9)$, linoleic acid $(18: 2 n-6)$ and total $n-6$ FA the values obtained for the individual and total FA using the weighed records tended to be higher than with the FFQ. For most FA there was no relationship between the difference in dietary intake assessed by the two dietary methods and the mean of the two estimates, the exception being eicosapentaenoic $(20: 5 n-3)$ and docosahexaenoic $(22: 6 n-3)$ acids, both of which demonstrated negative correlations (results not shown). Bias and limits of agreement, using ratio measures for $20: 5 n-3$ and $22: 6 n-3$ are shown in Table 2 .

Correlation coefficients comparing unadjusted FA measured by weighed record with those from the FFQ ranged from 0.29 for $18: 1 n-9$ to 0.71 for $20: 4 n-6$ (Table 2). Adjustment for total energy intake tended to increase the correlation coefficient for saturated FA and decrease it for the unsaturated FA.

\section{Discussion}

The present study shows that a simple, self-administered, semi-quantitative FFQ can provide useful and reliable estimates of dietary FA intake, with estimates of absolute intakes of the major dietary saturated and polyunsaturated FA obtained from the FFQ being similar to those obtained from a $7 \mathrm{~d}$ weighed record. We have used a $7 \mathrm{~d}$ weighed record as a gold standard, as opposed to other biological markers such as erythrocyte membrane or adipose tissue FA, as the aim of the study was to focus on measuring dietary intake accurately, and this outcome is not affected by metabolic processes. Although a few FFQ capable of estimating FA intake have been developed in other countries (Andersen et al. 1998; Wolk et al. 1998; Tokudome et al. 2001), none has been available previously in the UK. As a result, little epidemiological research has been done in the 


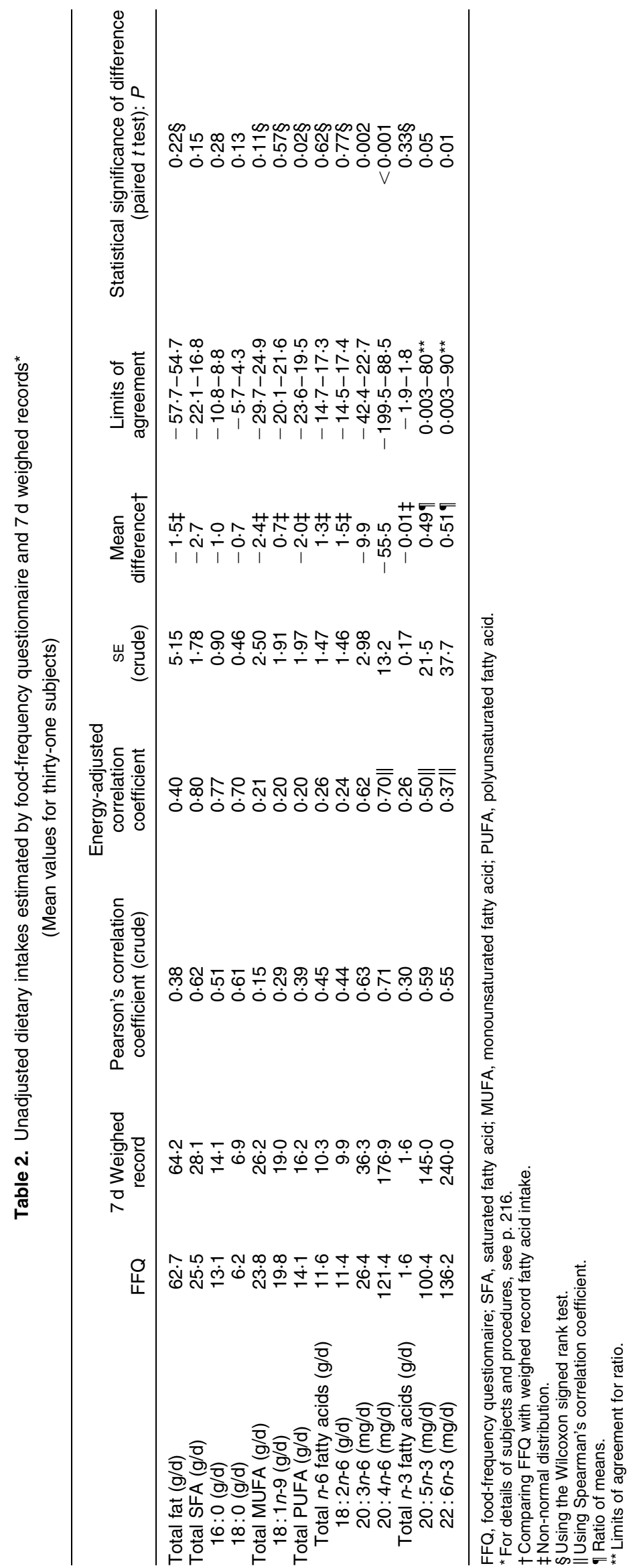


UK with regard to the intake of individual FA and disease, despite dietary fat intake being implicated in the aetiology of several diseases, including cardiovascular disease (Leaf \& Weber, 1988), breast cancer (Howe et al. 1990; Willett, 2001a) and inflammatory bowel disease (Geerling et al. 1998; Belluzzi et al. 2000).

The absolute mean FA intakes estimated by the two dietary assessment methods in the present study were similar, but because of the relatively small number of participants the agreement limits were wide. The maximum frequency option for intake of a food item in the FFQ was seven times per week, and clearly for some food items, such as fat spreads and drinks, the frequency may be as often as several times per $\mathrm{d}$. This may in part explain why the FA values tended to be higher for the weighed records than for the FFQ.

Our present study population was educated to a higher level than that of the general UK population and was of higher social class, an observation that is seen in other population studies of diet (Subar et al. 2001; Tokudome et al. 2001); this probably reflects the complex factors that motivate individuals to take part in research. Although the estimated saturated $(29.2 \mathrm{~g} / \mathrm{d})$, monounsaturated $(26.3 \mathrm{~g} / \mathrm{d})$ and polyunsaturated $(13.4 \mathrm{~g} / \mathrm{d})$ fat consumed by our present study participants was broadly similar to that of the average person in the UK National Food Survey in 2000, the total fat consumed by the study participants was lower (FFQ 62.7, $7 \mathrm{~d}$ weighed record $64.2 \mathrm{~g} / \mathrm{d}$ ) than that seen in the National Food Survey (74.0 g/d) (Ministry of Agriculture, Fisheries and Food, 2001a), suggesting that our present population may be more health and diet conscious than the general population. This is a well known problem with studies involving dietary assessments. Other factors which have been shown to effect diet and dietary recall include age (Campbell \& Dodds, 1967), gender (Campbell \& Dodds, 1967) and season (Hackett et al. 1985; de Castro, 1991); however, due to the sample size of the present study we were unable to assess the influence of these variables.

When completing the questionnaire, the participants were asked to consider their diet over the previous month, thereby providing an estimate of mean FA intake over this period. However, the participants completed only one $7 \mathrm{~d}$ weighed record, which will only provide an estimate of intake over that particular week and which may be less representative of their usual intake than that of the questionnaire. As the participants completed the two dietary assessment methods within 2 weeks of one another, it is unlikely that the participants' usual dietary habits would have been very different between completing the two assessments, and this would increase the likelihood of finding a higher correlation.

Unlike the FFQ, the weighed record does not depend upon memory, is open-ended and has direct portion measurement, but due to the recording intensity involved in keeping such a record is prone to both recording fatigue and adjustment of normal dietary intake. The weighed record is considered the most accurate method of dietary assessment, and as it also has the least correlated error with the FFQ it is considered to be the best dietary method to use when validating one (Willett, 1990b).

The FA databank used to calculate intake was obtained from the same source for both the weighed record and
FFQ, so we are unable to exclude some degree of systematic error. Data relating to the FA content of fruits and vegetables were limited, but although FA composition of these food items would be desirable, they contribute to only a small proportion of the total dietary fat consumed and are unlikely to make a significant difference to the FA intakes. Furthermore, data relating to the FA content of some of the higher-fat fruits, such as olives and avocados, were available and included in analyses of the dietary assessments.

There are differing opinions amongst nutritional epidemiologists as to the need to adjust nutrient intake for total energy intake (Block, 2001; Willett, 2001b), and we have therefore presented the results in both formats. However, if a specific nutrient is found to have an effect on disease outcome, one would wish to modify dietary intake of the nutrient without altering overall energy intake, as any long-term change in total energy intake without a corresponding change in energy expenditure will affect body weight. It is therefore due to this epidemiological interest in nutrient composition of the diet, rather than absolute intake, that we have provided the energy-adjusted results of comparisons between the dietary assessment methods.

The correlation coefficients between the FFQ and $7 \mathrm{~d}$ weighed record in our present study compare favourably with other similar studies both with regards to individual FA (crude mean $r$ 0.54, adjusted mean $r$ 0.51) (Wolk et al. 1998; Tokudome et al. 2001) and FA groups (crude mean $r$ 0.39, adjusted mean $r$ 0.38) (Willett et al. 1987; Jain et al. 1996; Andersen et al. 1998), and other more commonly measured micronutrients (Brunner et al. 2001). Unfortunately, $18: 1 n-9$ and total monounsaturated FA had poor correlation coefficients compared with the other FA in our present study and when compared with other studies (Jain et al. 1996; Wolk et al. 1998; Tokudome et al. 2001). This may in part be due to poor recording of the use of olive oil for cooking in the weighed records, as the mean intake was lower in the weighed records than the FFQ, and when this possibility was explored further not all those who had recorded regular use of olive oil for shallow frying in their FFQ recorded its use in the weighed record. Furthermore, olive oil was the most frequently used cooking oil in the present study but was not the only type used, and both total $n-6$ and $18: 2 n-6$, which are the main constituents of the majority of other cooking oils, also had lower reported intakes in the weighed records than the FFQ, supporting this theory.

It has been noted that crude correlation coefficients tend to improve when adjusted for energy intake (Willett \& Stampfer, 1986; Jain et al. 1996), as correlated measurement errors in energy intake and nutrient intake are effectively cancelled out. Although our results demonstrated this for saturated FA, we found the converse for polyunsaturated FA, and the reason for this is still unclear.

The composition of food is dynamic, and consequently as common food items become more or less popular and as the FA composition of food changes, it will be important to continue to update the food composition database and expand the database to include new products. The reasons for the poor correlation between the FFQ and weighed record $18: 1 n-9$ intakes will also need to be considered further and further refinement of the questionnaire may need to 
be made. In conclusion, although further work needs to be done on validating this questionnaire in a larger randomly selected group of the general population, this simple, selfadministered, semi-quantitative FFQ is capable of obtaining useful and reliable information about dietary FA intake in a selected UK population.

\section{Acknowledgements}

We thank Kirsten Whitehead and the Department of Dietetics and Nutrition at Nottingham City Hospital with their help and advice in adapting the FFQ, Tinuviel Software for customising the computer programmes used to analyse the data, The Institute of Brain Chemistry and Human Nutrition for the use of their food FA databank and the British Lung Foundation (grant no. P99/4) for their financial support.

\section{References}

Andersen LF, Solvoll K, Johansson LRK, Salminen I, Aro A \& Drevon CA (1998) Evaluation of a food frequency questionnaire with weighed records, fatty acids, and alpha-tocopherol in adipose tissue and serum. Am J Epidemiol 150, 75-87.

Belluzzi A, Boschi S, Brignola C, Munarini A, Cariani G \& Miglio F (2000) Polyunsaturated fatty acids and inflammatory bowel disease. Am J Clin Nutr 71, 339S-342S.

Berlin E, Bhathena SJ, McClure D \& Peters RC (1998) Dietary menhaden and corn oils and the red blood cell membrane lipid composition and fluidity in hyper- and normocholesterolemic miniature swine. J Nutr 128, 1421-1428.

Black PN (1999) The prevalence of allergic disease and linoleic acid. J Allergy Clin Immunol 103, 351-352.

Bland JM \& Altman DG (1986) Statistical methods for assessing agreement between two methods of clinical measurement. Lancet 1, 307-310.

Block G (2001) Invited commentary: another perspective on food frequency questionnaires. Am J Epidemiol 154, 1103-1104.

Broadfield E, McKeever TM, Scrivener S, Venn A, Lewis SA \& Britton J (2002) Increase in the prevalence of allergen skin sensitization in successive birth cohorts. J Allergy Clin Immunol 109, 969-974.

Brunner E, Stallone D, Juneja M, Bingham S \& Marmot M (2001) Dietary assessment in Whitehall II: comparison of $7 \mathrm{~d}$ diet diary and food-frequency questionnaire and validity against biomarkers. Br J Nutr 86, 405-414.

Burr ML, Fehily AM, Rogers S, Welsby E, King S \& Sandham S (1989) Diet and reinfarction trial (DART): design, recruitment and compliance. Eur Heart J 10, 558-567.

Campbell VA \& Dodds ML (1967) Collecting dietary information from groups of older people. J Am Dietet Assoc 51, 29-33.

Crawley H (1994) Food Portion Sizes. London: H. M. Stationery Office.

de Castro JM (1991) Seasonal rhythms of human nutrient intake and meal pattern. Physiol Behav 50, 243-248.

Endres S, Ghorbani R, Kelley VE, et al. (1989) The effect of dietary supplementation with $n-3$ polyunsaturated fatty acids on the synthesis of interleukin-1 and tumor necrosis factor by mononuclear cells. New Eng J Med 320, 265-270.

Fogarty A \& Britton J (2000) The role of diet in the aetiology of asthma. Clin Exp Allergy 30, 615-627.

Geerling BJ, Houwelingen AC, Badart-Smook A, Stockbrügger RW \& Brummer R-J (1998) Fat intake and fatty acid profile in plasma phospholipids and adipose tissue in patients with Crohn's disease, compared with controls. Am J Gastroenterol 94, 410-417.
Hackett AF, Appleton DR, Rugg-Gunn AJ \& Eastoe JE (1985) Some influences on the measurement of food intake during a dietary survey of adolescents. Hum Nutr Appl Nutr 39, $167-177$.

Holland B, Welch AA, Unwin ID, Buss DH, Paul AA \& Southgate DAT (1991) McCance and Widdowson's The Composition of Foods, 5th ed. Cambridge: Royal Society of Chemistry \& Ministry of Agriculture, Fisheries and Food.

Horrobin DF (1987) Low prevalences of coronary heart disease (CHD), psoriasis, asthma and rheumatoid arthritis in Eskimos: are they caused by high dietary intake of eicosapentaenoic acid (EPA), a genetic variation of essential fatty acid (EFA) metabolism or a combination of both? Med Hypotheses 22, 421-428.

Howe GR, Hirohata T, Hislop TG, et al. (1990) Dietary factors and risk of breast cancer: combined analysis of 12 case-control studies. J Natl Cancer Inst 82, 561-569.

Jain M, Howe GR \& Rohan T (1996) Dietary assessment in epidemiology: a comparison of a food frequency and a diet history questionnaire with a 7-day food record. Am J Epidemiol 143, 953-959.

Leaf A \& Weber PC (1988) Cardiovascular effects of $n-3$ fatty acids. New Eng J Med 318, 549-557.

Lee TH \& Austen KF (1986) Arachidonic acid metabolism by the 5-lipoxygenase pathway, and the effects of alternative dietary fatty acids. Adv Immunol 39, 145-175.

Ministry of Agriculture, Fisheries and Food (2001a) Household nutrient data from 1940 onwards. http://www.maff.gov.uk/esg/ default.htm

Ministry of Agriculture, Fisheries and Food (2001b) Household consumption of selected foods from 1942 onwards. http://www. maff.gov.uk/esg/default.htm

Schwartz J (2000) Role of polyunsaturated fatty acids in lung disease. Am J Clin Nutr 71, 393S-396S.

Simopoulos AP (1996) The role of fatty acids in gene expression: health implications. Ann Nutr Metabol 40, 303-311.

Subar AF, Thompson FE, Kipnis V, et al. (2001) Comparative validation of the Block, Willett, and National Cancer Institute food frequency questionnaires: The Eating at America's Table Study. Am J Epidemiol 154, 1089-1099.

Tokudome S, Imaeda N, Tokudome Y, et al. (2001) Relative validity of a semi-quantitative food frequency questionnaire versus 28 day weighed diet records in Japanese female dieticians. Eur $J$ Clin Nutr 55, 735-742.

Vollmer WM, Osborne ML \& Buist AS (1998) 20-year trends in the prevalence of asthma and chronic airflow obstruction in an HMO. Am J Respir Crit Care Med 157, 1079-1084.

Willett WC (1990a) Implications of total energy intake for epidemiological analyses. In Nutritional Epidemiology, pp. 245-271. New York: Oxford University Press.

Willett WC (1990b) Reproducibility and validity of food-frequency questionnaires. In Nutritional Epidemiology, pp. 92-126. New York: Oxford University Press.

Willett WC (2001a) Diet and breast cancer. J Intern Med 249, $395-411$.

Willett WC (2001b) Invited commentary: A further look at dietary questionnaire validation. Am J Epidemiol 154, 1100-1102.

Willett WC, Reynolds RD, Cottrell-Hoehner S, Sampson LA \& Browne ML (1987) Validation of a semi-quantitative food frequency questionnaire: Comparison with a 1-year diet record. J Am Diet Assoc 87, 43-47.

Willett WC \& Stampfer MJ (1986) Total energy intake: implications for epidemiologic analyses. Am J Epidemiol 124, 17-27.

Wolk A, Ljung H, Vessby B, Hunter D \& Willett WC (1998) Effect of additional questions about fat on the validity of fat estimates from a food frequency questionnaire. Eur J Clin Nutr 52, 186-192. 